\title{
The shape of the surface of a rotating mass of water as a variational problem
}

\author{
A forma da superfície de uma massa de água girante como um problema variacional \\ F.C. Santos, A.C. Tort* \\ Universidade Federal do Rio de Janeiro, Rio de Janeiro, RJ, Brazil
}

Received on September 13, 2016. Accepted on September 24, 2016.

\begin{abstract}
Variational methods have a long and remarkable role in theoretical physics. Few of our students when first exposed to them fail to admire their elegance and efficacy in the formulation and solution of physical problems. In this paper we apply the variational approach that leads to the Euler-Lagrange equations to the determination of the shape of the surface of a mass of water that partially fills a cylindrical bucket that rotates with constant angular velocity (Newton's bucket). Here this approach will lead us to the principle of minimization of the effective potential energy associated with the system. The effect of an external pressure on the equilibrium shape is also taken into account and two models, the constant pressure model and the linear model are discussed. The level of the discussion is kept accessible to undergraduates taking an intermediate level course in classical mechanics.
\end{abstract}

Keywords: analytical mechanics, variational calculus, rotating bucket.

Métodos variacionais têm um longo e destacado papel na física teórica. Poucos são os nossos estudantes que quando apresentados pela primeira vez a estes métodos não são tomados de admiração por sua elegância e eficiência na formulação e solução de problemas físicos. Neste trabalho fazemos uso da abordagem variacional que leva às equações de Euler-Lagrange na determinação da forma da superfície de uma massa de água que preenche parcialmente um recipiente cilíndrico que gira com velocidade angular constante (o balde de Newton). Aqui, essa abordagem nos levará ao princípio de minimização da energia potencial efetiva associada com o sistema. O efeito de uma pressão externa sobre sobre a forma de equilíbrio também é levado em conta e dois modelos, o modelo da pressão constante e o modelo linear são discutidos. O nível da discussão é acessível aos estudantes de graduação inscritos em um curso de mecânica clássica de nível intermediário.

Palavras-chave: Mecânica analítica, cálculo variacional, balde girante.

\section{The variational approach: A simple example}

We begin by illustrating the variational approach with a simple example: The determination of the shape of the free surface of liquid contained in a uniformly accelerated vessel, see Figure 1. The applied forces that can be associated with a potential energy function are the weight and the atmospheric force which by its turn is associated with atmospheric pressure. If we suppose that the atmospheric pressure is uniform and the liquid incompressible the

*Endereço de correspondência: tort@if.ufrj.br

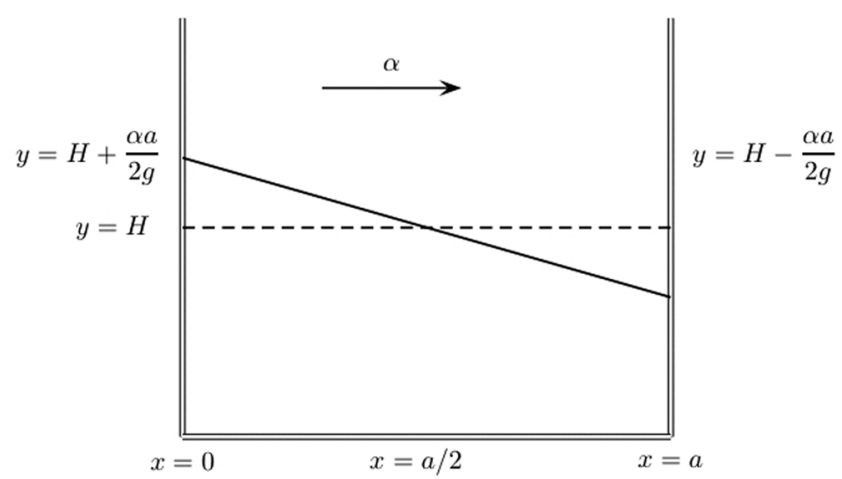

Figure 1: A linear accelerated mass of water. The inclined free surface of the water intercepts the leveled surface at $x=a / 2$. 
work done by atmospheric force during the deformation of the liquid is null,

$$
W_{\text {atmosphere }}=\int p_{\text {atmospheric }} d v=0 .
$$

On the other hand, in the non-inertial frame attached to the accelerated vessel where the liquid is at rest there is a field $-\boldsymbol{\alpha}$ where $\boldsymbol{\alpha}$ is the vessel's acceleration. Therefore, there are two contributions to the effective potential energy, the gravitational potential energy and the non-inertial potential energy. Let us suppose that the vessel has a retangular bottom whose sides are $a$ and $b$. The height of the vessel is $H$, hence the mass of the liquid is $M=\rho a b H$, where $\rho$ is the density of the liquid. Let us introduce coordinates $x, y$, and $z$ attached to the vessel with the origem placed at the lower left point and write for an element of mass $d M$ the differential potential energy

$$
d U_{\text {gravitational }}=d M g z=\rho g z d x d y d z
$$

which upon integrations reads

$$
\begin{aligned}
U_{\text {gravitational }} & =\rho g \int_{0}^{a} d x \int_{0}^{b} d y \int_{0}^{f(x)} z d z \\
& =\rho g b \int_{0}^{a} \frac{[f(x)]^{2}}{2} d x,
\end{aligned}
$$

where $f(x)$ is the shape of the free surface of the liquid that we wish to determine. The differential of the potential energy associated with the inertial force is

$$
d U_{\text {inertial }}=d M \alpha x=\rho \alpha x d x d y d z .
$$

Integrating this expression we obtain

$$
\begin{aligned}
U_{\text {inertial }} & =\rho \alpha \int_{0}^{a} x d x \int_{0}^{b} d y \int_{0}^{f(x)} z d z \\
& =\rho \alpha b \int_{0}^{a} x f(x) d x .
\end{aligned}
$$

The effective potential energy $U_{\text {effective }}=U_{\text {gravitational }}+$ $U_{\text {inertial }}$ reads

$$
U_{\text {effective }}=\rho b \int_{0}^{a}\left(\frac{g}{2}[f(x)]^{2}+\alpha x f(x)\right) d x .
$$

The constraint is given by the condition that the mass of water must be constant. However, if the liquid is supposed to be incompressible we can state also that its volume must be constant, that is

$$
\begin{aligned}
V & =a b H=\int_{0}^{a} d x \int_{0}^{b} d y \int_{0}^{f(x)} d z \\
& =b \int_{0}^{a} f(x) d x .
\end{aligned}
$$

As we will see below this will allow us to identify more easily the constraint forces. Before we proceed with the determination of $f(x)$ let us recall briefly the variational approach to this type of problem.

Consider the functional

$$
I[f]=\int_{x_{a}}^{x_{b}} F[f(x) ; x] d x ;
$$

where $f(x)$ has at least a continuos first order derivative. The function $f(x)$ that extremizes the functional $I[f]$ must satisfy the condition

$$
\begin{aligned}
\delta I[f] & =\int_{x_{a}}^{x_{b}} F[f(x)+\eta(x) ; x] d x \\
& -\int_{x_{a}}^{x_{b}} F[f(x) ; x] d x=0 ;
\end{aligned}
$$

where $\eta(x)$ is an arbitrarily small perturbation added to $f(x)$ at each point $x \in\left[x_{a}, x_{b}\right]$ that satisfies the conditions

$$
\eta\left(x_{a}\right)=\eta\left(x_{b}\right)=0 .
$$

If we expand equation (9) we obtain after an integration by parts

$$
\delta I[f]=\int_{x_{a}}^{x_{b}} \frac{\partial F}{\partial f} \eta(x) d x=0 .
$$

Since $\eta(x)$ is an arbitrary function it follows that

$$
\frac{\partial F}{\partial f}=0
$$

which is a simplified form of the Euler-Lagrange equation. Suppose now that we add a constraint condition given by

$$
\int_{x_{a}}^{x_{b}} G[f(x), x] d x=\text { constant. }
$$

Because this constant does not affect the EulerLagrange equation we are looking for we can set it equal to zero. Following [2] we introduce the Lagrange multiplier $\lambda$ and write

$$
I^{\prime}[f]=\int_{x_{a}}^{x_{b}} F[f(x), x] d x+\lambda \int_{x_{a}}^{x_{b}} G[f(x), x] d x .
$$


Setting $\delta I^{\prime}[f]=0$ and proceeding as before we obtain

$$
\frac{\partial F}{\partial f}+\lambda \frac{\partial G}{\partial f}=0
$$

This particular form of the Euler-Lagrange equation is sufficient to our purposes here. A more general approach leads the standard form of the EulerLagrange equation

$$
\frac{\partial F}{\partial f}-\frac{d}{d x} \frac{\partial F}{\partial f^{\prime}}=0
$$

where now $F\left[f(x), f^{\prime}(x), x\right]$, see $[2$. It can be shown that we can replace Cartesian coordinates for more suitable generalized ones without modifying the form of the Euler-Lagrange equation [2] .

Going back to our problem we see that

$$
F[f(x), x]=b\left(\frac{\rho g}{2}[f(x)]^{2}+\rho \alpha x f(x)+\lambda f(x)\right),
$$

where $\lambda$ is the Lagrange multiplier. Taking equation (17) into equation 15 we obtain

$$
f(x)=-\frac{\alpha}{g} x-\frac{\lambda}{\rho g},
$$

and taking this result into equation (7) we find

$$
\lambda=-\frac{\rho \alpha a}{2}-\rho g H .
$$

The Lagrange multiplier can be interpreted as the mean constraint forces per unit area on the surfaces on which they are applied. Therefore,

$$
f(x)=-\frac{\alpha}{g} x+\frac{\alpha a}{2 g}+H .
$$

Equation 20 represents a straight line with a negative slope that intercepts the vessel walls at $x=0$ and $x=a$. At $x=a / 2$ it intercepts the leveled free surface of the water $y=H$, see Figure 1 .

\section{The variational approach: The rotating bucket}

Consider now a vessel in the shape of a right cylinder the radius of which is $R$ partially filled with water or some other liquid with uniform density $\rho$ up to a certain height $H$. Suppose that the container starts to rotate with constant angular velocity $\boldsymbol{\omega}$ about the symmetry axis perpendicular to the bottom of the container and passing through its geometrical center. From now on we will label this axis as the $z$ axis, see Figure 2. Suppose also for the moment that the mass of water and the bucket rotate in vacuo. The water initially remains at rest but little by little it starts to move and ends up rotating conjointly with the bucket. As the water begins to move, its surface starts to curve until it attains its final shape. Our problem will be to determine analytically the shape of this surface taking into account that the volume or if we prefer the mass of the water must remain constant. The physical interpretation of this gedanken experiment plays an important role in Newton's conception of (absolute) space [1].

$$
d U_{\text {gravitational }}=d M g z=\rho d V g z=\rho r d r d \phi d z g z,
$$

where we are using cylindrical coordinates $r, \phi$ and $z$ with the origin at the geometrical center of the bottom of the bucket and hence $r$ is the perpendicular distance to the rotation axis $z$. The total gravitational potential energy is

$$
\begin{aligned}
U_{\text {gravitational }} & =\rho g \int_{0}^{2 \pi} d \phi \int_{0}^{R}\left[\int_{0}^{f(r)} z d z\right] r d r \\
& =\pi \rho g \int_{0}^{R}[f(r)]^{2} r d r
\end{aligned}
$$

where $f(r)$ is the height of the water surface at a distance $r$ from the $z$ axis and defines its shape. Now we evaluate the centrifugal potential energy. For an element of mass $d M$

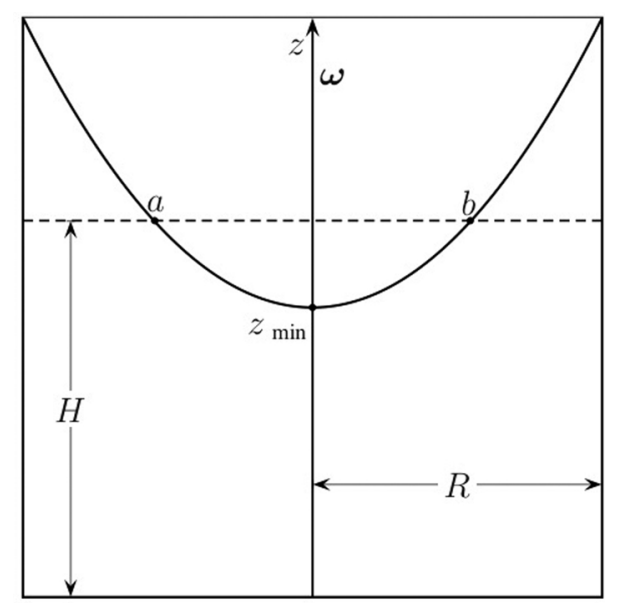

Figure 2: A rotating mass of water constrained by a cylindrical bucket. The dashed line at $z_{\text {surface }}=H$ indicates the surface of the water when $\boldsymbol{\omega}=0$. 


$$
d U_{\text {centrifugal }}=-\frac{1}{2} d M \omega^{2} r^{2}=-\frac{1}{2} \rho r d r d \phi d z \omega^{2} r^{2}
$$

Therefore the total centrifugal potential energy is

$$
U_{\text {centrifugal }}=-\frac{1}{2} \rho \omega^{2} \int_{0}^{2 \pi} d \phi \int_{0}^{R}\left[\int_{0}^{f(r)} d z\right] r^{3} d r
$$

or

$$
U_{\text {centrifugal }}=-\rho \pi \omega^{2} \int_{0}^{R} f(r) r^{3} d r
$$

The constraint is given by the mass $M$ of the water which must be constant

$$
M=\int \rho d V=\text { constant }
$$

If we suppose as before that the mass of water remains incompressible we can also write

$$
\begin{aligned}
V & =\int_{0}^{2 \pi} d \phi \int_{0}^{R}\left[\int_{0}^{f(r)} d z\right] r d r \\
& =2 \pi \int_{0}^{R} f(r) r d r .
\end{aligned}
$$

The complete functional to be extremized is

$$
U_{\text {effective }}=U_{\text {centrifugal }}+U_{\text {gravitational }}+\lambda V
$$

where $\lambda$ is the Lagrange undetermined multiplier. Making use of equations (22), 25) and (26) we obtain

$$
U_{\text {effective }}[f]=\int_{0}^{R} F[f(r), r] d r
$$

where

$$
\begin{aligned}
F[f(r), r] & =\pi \rho\left[-\omega^{2} f(r) r^{3}+g[f(r)]^{2} r\right. \\
& \left.+2 \frac{\lambda}{\rho} f(r) r\right]
\end{aligned}
$$

If impose the condition $\delta U[f]=0$, we are led to the Euler-Lagrange equation (15) and It follows that

$$
\frac{\partial F}{\partial f}=-\omega^{2} r^{2}+2 g f(r)+2 \lambda=0
$$

or

$$
f(r)=\frac{\omega^{2}}{2 g} r^{2}-\frac{\lambda}{\rho g}
$$

We now evaluate the Lagrange multiplier. Taking equation (31) into equation (26), we obtain

$$
V=2\left(\frac{\omega^{2} R^{4}}{8 g}-\frac{\lambda R^{2}}{2 \rho g}\right) .
$$

Remembering that $V=\pi R^{2} H$ it follows that

$$
\lambda=\frac{\rho \omega^{2} R^{2}}{4}-\rho g H
$$

and we finally obtain

$$
\begin{array}{r}
z_{\text {superface }}=f(r)=\frac{\omega^{2}}{2 g}\left(r^{2}-\frac{R^{2}}{2}\right)+H, \\
0 \leq r \leq R .
\end{array}
$$

Equation (34) represents an inverted paraboloid of revolution and it also defines a surface of constant effective potential energy. It is easy to verify that

$$
\begin{gathered}
\omega=0, \quad z_{\text {superface }}=H, \quad \forall r \in[0, R] ; \\
\omega \neq 0, \quad z_{\text {surface }}=H, \quad \text { for } \quad r=\frac{R}{\sqrt{2}}
\end{gathered}
$$

points $a$ and $b$ in Figure 2 .

$$
\begin{gathered}
\omega \neq 0, \quad z_{\text {surface }}>H, \quad \text { for } \quad r>\frac{R}{\sqrt{2}} ; \\
\omega \neq 0, \quad z_{\text {surface }}<H, \text { for } r<\frac{R}{\sqrt{2}} . \\
\omega \neq 0, \quad z_{\text {surface } \min }=-\frac{\omega^{2} R^{2}}{4 g}+H, \quad \text { for } \quad r=0 . \\
\omega \neq 0, \quad z_{\text {surface } \max }=+\frac{\omega^{2} R^{2}}{4 g}+H, \quad \text { for } \quad r=R .
\end{gathered}
$$

If we consider two points on the parabolic surface with $r \in[0, R]$ and a straight line connecting these two points we see that the straight line lies above the graph of $f(r)$ hence the surface is concave upwards or convex. It is clear that gravity is a critical feature in what concerns the final shape of the surface of the water in the stationary rotational state of the system. It is convenient to write equation (34) in the non-dimensional form

$$
z_{\text {surface }}^{*}=\frac{a_{0}}{g}\left(\frac{x^{2}}{2}-\frac{x_{R}^{2}}{4}\right)+1, \quad x \in[0,1],
$$


where $z_{\text {surface }}^{*}=z_{\text {superface }} / H, x=r / H, x_{R}=R / H$, and $a_{0}=\omega^{2} H$. The minimum value for $z_{\text {surface }}^{*}$ corresponds to $x=0$, that is

$$
z_{\text {surface, } \min }^{*}=-\frac{a_{0}}{g} \frac{x_{R}^{2}}{4}+1 \geq 0 .
$$

From this it follows that $z_{\text {surface, min }}^{*}=0$ when

$$
\frac{a_{0}}{g}=\frac{4}{x_{R}}
$$

Figure 3 shows the parabolic surface for several values of $a_{0} / g$. As this quantity increases, $z_{\text {surface, } \min }^{*} \rightarrow$ 0 until it hits the bottom of the bucket. From this point on equation (35) leads to negative values of $z_{\text {surface, } \min }^{*}$ and though mathematically sound negative values here mean that they are below the bottom of the bucket.

\section{The effect of an external pressure}

The effect of an external pressure, e.g. the atmospheric pressure, can be taken into account by adding to the effective potential energy a term that represents the contribution of the atmospheric pressure to the deformation of the surface of the water. For an infinitesimal deformation this contribution reads

$$
d U_{\text {atm }}=p_{\text {atm }} r d r d \phi d z .
$$

Integrating this contribution we have

$$
U_{\mathrm{atm}}=2 \pi \int_{H}^{R} \int_{0}^{f(r)} p(z) d z r d r .
$$

where $p(z)$ is the pressure which we will assume to be dependent only on $z \geq 0$. Assuming that the liquid is incompressible as we did before the effective potential now reads

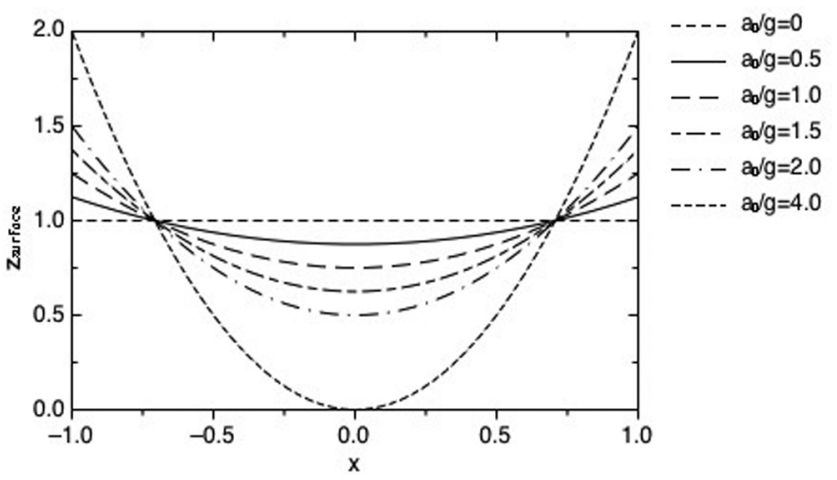

Figure 3: Parabolic surfaces for $x_{R}=1$ and several values of $a_{0} / g$.

$$
\begin{aligned}
U_{\text {eff }} & =\rho \pi \int_{H}^{R}\left[-\omega r^{2} r^{3}+g[f(r)]^{2} r\right. \\
& \left.+2 \frac{\lambda}{\rho} r+2 \frac{r}{\rho} \int_{0}^{f(r)} p(z) d z\right] d r .
\end{aligned}
$$

The Euler-Lagrange equation then leads to

$$
f(r)+\frac{1}{\rho g} p[f(r)]=\frac{\omega^{2} r^{2}}{2 g}-\frac{\lambda}{\rho g} .
$$

If the pressure $p[f(r)]$ is known we can solve for $f(r)$ and making use of the constraint, equation (26), determine the Lagrange multiplier $\lambda$. Substituting $\lambda$ into equation (41) we finally express the function $f(r)$ explicitly without undetermined constants. Now we take this $f(r)$ into equation 28 )

$$
\lambda=\frac{\rho \omega^{2} R^{2}}{4}-\rho g H-\frac{2}{R^{2}} \int_{0}^{R} p[f(r)] r d r .
$$

This form is convenient for the interpretation of the Lagrange mulltiplier. The first term on the RHS of equation (42) corresponds to the to the mean force per unit area on the lateral surface of the liquid, and the second and third termos taken together correspond to to the mean total force per unit area on the botton of the liquid.

\subsection{Uniform pressure model}

A simple model is to suppose that the pressure is uniform, that is $p[f(r)]=p_{0}$, then equations (41) and (42) lead to

$$
f(r)+\frac{p_{0}}{\rho g}=\frac{\omega^{2} r^{2}}{2 g}-\frac{\lambda}{\rho g},
$$

and (42) yields the result given by equation (34) showing that a constant pressure does not contribute to the final shape of the free surface of the water though the effect of the external pressure shows itself in the Lagrangian multiplier which is an indication of the action of constraint forces.

\subsection{Linear model}

A less simple model is the linear one $p(z)=p_{0}+\gamma z$, where $\gamma$ is a constant with the appropriate dimensions. Adopting a linear model means that

$$
p[f(r)]=p_{0}+\gamma f(r) .
$$


Taking this expression into equation 41 we obtain

$$
f(r)=\frac{1}{\rho g}\left(p_{0}+\gamma f(r)\right)=\frac{\omega^{2} r^{2}}{2 g}-\frac{\lambda}{\rho g},
$$

and regrouping the terms in equation 45 we obtain

$$
f(r)+\frac{p_{0}}{\rho g\left(1+\frac{\gamma}{\rho g}\right)}=\frac{\omega^{2} r^{2}}{2 g\left(1+\frac{\gamma}{\rho g}\right)}-\frac{\lambda}{\rho g} .
$$

Comparing equations (46) and (43) we see that a linear variation of the pressure is equivalent to a constant pressure with an effective gravity given by

$$
g_{\mathrm{eff}}=g\left(1+\frac{\gamma}{\rho g}\right)
$$

Therefore the complete solution of the linear model amounts to replacing $g$ by $g_{\text {eff }}$ into equation (43) and to evaluating $\lambda$ with equation 42 with $p=p_{0}$, hence

$$
\lambda=\frac{\rho \omega^{2} R^{2}}{4}-\rho g_{\mathrm{eff}} H-p_{0}
$$

and

$$
f(r)=\frac{\omega^{2}}{2 g_{\mathrm{eff}}}\left(r^{2}-\frac{R^{2}}{2}\right)+H, \quad 0 \leq r \leq R
$$

The free surface of the rotating mass of water is still parabolic but determined by an effective gravitational acceleration. We see also that an increase/decrease in $\gamma$ gives rise to an increase/ decrease of the effective gravitational acceleration thus affecting the parabolic equilibrium shape. Notice that since $f(r)>0$ in the closed interval $[0, R]$ and the dimensionless factor $\gamma / \rho g_{\text {eff }}$ is also $>0$, it follows that the maximum value of $f(r)$ is greater when compared with the constant pressure or pressureless cases.

\section{Final remarks}

The functionals that we have made use here, equations (6), 29), 40), do not depend on the derivatives of the shape function $f$ hence the Euler-Lagrange equation is reduced to a constraint and does not depend on arbitrary constants. In principle, the role of the atmospheric pressure is negligible but nothing can prevent us of imagining environments where this may not be so, for instance some super Earth type of exoplanet. To conclude, the final shape of the surface of a rotating mass of water constrained by a co-rotating bucket as analyzed in the co-rotating frame is a compromise between the centrifugal effects and gravity and if we add an external pressure it becomes a compromise between the former two and the latter. Both examples show the usefulness and elegance of the variational approach to physical problems.

\section{References}

[1] I. Newton, The Principia (Prometheus, Amherst, 1995), translated by Andrew Motte.

[2] M. Levi, Classical Mechanics with Calculus of Variations and Optimal Control (American Mathematical Society, Providence, 2014). 